\title{
LA MILICIANA ADA Y LA DINÁMICA DE PODER EN LA LITERATURA AFRICANA HISPANÓFONA
}

JOANNA BOAMPONG

Universidad de Ghana

jboampong@ug.edu.gh

\section{RESUMEN}

La novela, Los poderes de la tempestad de Donato Ndongo permite el estudio de las relaciones de poder desde distintos ángulos sean las que pueden manifestarse entre régimen militar y ciudadanos, ciudadanos y extranjeros, alfabetizados y analfabetos, hombres y mujeres.En este trabajo pretendemos evaluar la compleja situación de una mujer que parece estar dotada de gran parte de los elementos tradicionalmente reservados para el hombre como son el poder, la voz y la agencia. ¿Hasta qué punto el poder puede asociarse con una mujer en una sociedad postcolonial africana tan represiva y patriarcal, como la que se presenta en la obra? Por su labor profesional, posición, desempeño y discurso, la miliciana Ada se sitúa fuera de la esfera tradicional acordada a la mujer, y cobra cierto poder, agencia y voz. Así, simboliza el logro de algunos de los derechos quizás más importantes que se reclaman para la mujer africana. Aun así, la pregunta que cabe hacer es la siguiente: ¿acaso deja de ser víctima la mujer aunque se ubique en el espacio del hombre y esté dotada de "privilegios" tradicionalmente reservados para los hombres?

PAlabRas Clave: mujer, voz, poder, sexualidad, espacio, víctima.

\section{ABSTRACT}

Donato Ndongo's Los poderes de la tempestad makes it possible to examine power relations from different angles be they those that are manifested between a military regime and its citizens, citizens and foreigners, literates and illiterates, men and women. In this paper, we seek to evaluate the complex situation of a woman who appears to be endowed 
with elements traditionally reserved for men such as power, voice, and agency. To what extent can power be associated with a woman in a postcolonial society as repressive and patriarchal as the one presented in the work? On account of her profession, position, actions and discourse, Ada, the militia woman is positioned outside the traditional sphere conceded to the woman, and attains a level of power, agency and voice. Thus, she symbolizes the achievement of perhaps some of the most important rights sought for the African woman. However, considering the dynamics of power that play out in the novel, the question that remains is: does the woman cease to be a victim even though she is positioned in the man's space and endowed with "privileges" traditionally reserved for men?

KEY WoRDS: woman, voice, power, sexuality, space, victim.

\section{INTRODUCCIÓN}

Las sociedades africanas tales como se ven exhibidas en su tradición literaria se caracterizan por un patriarcalismo que se torna aún más intenso cuando hay conflicto, inestabilidad política o regímenes represivos. Como ya se ha establecido, en estos sistemas patriarcales, se le concede a la mujer una posición subordinada y pasiva, se le asigna el espacio específico del hogar, el mercado o la finca y su papel es el de proveer cariño y comida a la familia, ser madre o hija complaciente. Aunque este papel que desempeña la mujer es importante e incluso fundamental, no implica el poder. Como bien afirma Ali Mazrui:

In real life, motherhood leaves the African woman at the center but not necessarily in power. Woman has also been at the center of the economy, of water supply, and of energy supply in rural Africa without ever being powerful. This situation is reflected in the legend of the African woman as custodian of fire, custodian of earth, and custodian of water; woman at the center but not empowered (100-101).

Reitera esta afirmación Meyre Ivone da Silva (131) cuando señala que "An African woman is frequently seen as fertile mother of the nation, an image that African male writers have contributed to disseminating". En Los poderes de la tempestad, de Donato Ndongo se siguen hasta cierto punto los patrones de esta ideología. La mayoría de las mujeres que pueblan el relato se ven en posiciones y condiciones tradicionales arriba señaladas como son los casos de: Ángeles (mujer del protagonista central, madre de Rut); Avomo (mujer de Mbo, rodeada de chiquillos); Clo (joven con quien el protagonista tiene una aventura y quien se ocupa de traerle comida cuando está encarcelado). Se sitúan en un entorno íntimo de la casa, cumpliendo funciones más bien mundanas fuera de la óptica pública. La existencia de cada una de ellas es en función del papel que ejerce en la vida de otros-Mbo, los chiquillos, el protagonista no nombrado, Rut, etc. Ninguna de ellas es independiente, ninguna juega un papel determinante en los asuntos narrados en la novela, ni participa en la esfera pública, excepto Ada la miliciana. La pregunta que surge, y que nos proponemos examinar en este estudio es simplemente la siguiente: ¿Acaso, que una mujer tenga voz, agencia, y sea independiente implica que haya superado su posición de marginalizada o víctima para acceder al poder? 
La miliciana Ada se presenta solamente tres veces en Los poderes de la tempestad (1997), segunda novela en la trilogía "Los hijos de la tribu" de Donato Ndongo". Sin embargo, aunque se puede considerar a Ada como uno de los personajes secundarios, su presentación permite plantear cuestiones importantes en lo tocante a asuntos de poder y la política del feminismo en África. Un bosquejo de las obras que componen la literatura hispanófona, revela el hecho de que Ada es una protagonista única y ocupa un lugar exclusivo en el desarrollo de las protagonistas femeninas. ${ }^{2}$ Como bien afirma Daniel Grier, "Prácticamente la única mujer con cierto protagonismo en la novela es la militar, Ada, amenazante presencia, mujer hipersexuada y brutal que literalmente es una mujer de pelo en pecho, ya que tiene pelos 'como alambres' en los pezones." (74). A diferencia de todas las mujeres en la novela que anteriormente nombramos, nunca se presenta a Ada dentro de un entorno "tradicional" de la mujer africana. Presenciamos a Ada primero en su trabajo como miliciana en el aeropuerto, partícipe activa en el registro de pasajeros recién llegados del extranjero, y en el caos total que allí rige. Luego la encontramos en la calle, lugar que simboliza el desenfreno (y el libertinaje) en sus distintas formas. Y, finalmente, la vemos en la cárcel de Blavis donde ocurren algunas de las brutalidades más viciosas de la novela. No se le asocia de ningún modo con el entorno doméstico dentro del cual se suelen ubicar a los personajes femeninos africanos. Al contrario, su trabajo de militar la sitúa dentro de un ambiente público tradicionalmente reservado a los hombres. Y, es más, forma parte de los milicianos del presidente Macías, el equipo gubernamental encargado de poner en marcha su política tan importante como la re-africanización del país y la erradicación de todos los vestigios del recién abolido colonialismo español.

Si consideramos el hecho de que la mujer, en el sistema tradicional (re-africanizado) que se busca re-establecer, ni tiene derecho a tomar decisiones que le afectan a ella misma, caso paradigmático que encontramos en Ekomo de María Nsüe Angüe cuando Nnanga afirma que: "La vida de una mujer está siempre expuesta a las decisiones del abáa desde que nace hasta que muere (239-240), la posición que ocupa Ada, podría implicar un logro importante para la mujer. A diferencia de Nnanga, que según afirma Mbare Ngom (417) es "mujer fiel en la búsqueda de su identidad como ser humano y como mujer y, por ende, de su libertad en un universo que le impone muchas restricciones"3, Ada parece disfrutar de libertades que ni el protagonista central de Los poderes de la tempestad, ni la mayoría de los ciudadanos retratados en la novela tienen. Sin lugar a dudas esto se hace posible, en parte, por la profesión de miliciana que Ada ejerce.

Recordemos la siguiente afirmación de Ali Mazrui que arroja luz sobre una instancia que hace posible la libertad para la mujer africana: "Female liberation in Africa, but not necessarily female empowerment, sometimes gains from military rule" (100). Como mili-

${ }^{1}$ La primera vez es en el capítulo uno, páginas 23-27; el segundo encuentro ocurre en el capítulo cuatro, páginas 95-96, y finalmente en el capítulo diez, páginas 255-261.

${ }^{2}$ Nnanga en Ekomo (1985), Antígona en Antígona (1991), Bea en Bea (2000), Eldania en El llanto de la perra (2005), Anne Mengue en El metro (2007), María Fatima en Las tres virgenes de Santo Tomás (2008), por mencionar solo unas, son algunas protagonistas femeninas notables en la literatura africana hispanófona.

3 Citado en Mendogo, Minsongui Dieudonné. "Mujer y creación literaria en Guinea Ecuatorial”, EPOS, n. ${ }^{\circ} 13$, 1997, pp. 209-218. 
ciana, en un régimen autocrático Ada parece encarnar la liberación femenina a la que hace referencia Mazrui. Sin embargo, más allá de la libertad, y como discutimos a continuación, parece disfrutar del poder que según lo arriba citado se le suele negar a la mujer. Por ende, su participación en la gestión del país marca un desarrollo en el tratamiento de la mujer en la novela ecuatoguineana y es simbólico del cumplimiento de algunos de los derechos por los que lucha el feminismo africano. Me refiero al concepto de Social Transformation Including Women in Africa, la transformación social que incluye a las mujeres en África, y de cuyas siglas se deriva el concepto STIWANISM ${ }^{4}$. Como miliciana, Ada participa en la esfera pública y desde esta posición puede contribuir a la construcción del país y/o su transformación (para bien o mal).

Además, como militar Ada tiene la obligación de cumplir con lo que dicta el jefe de estado, que también es jefe del ejército. Debe trasmitir los deseos del jefe de estado a sus conciudadanos y poner en práctica cualquier política que busca implementar. De este modo Ada hace visible y patente la presencia latente del dictador. Ada es su agente, lo representa, da forma a sus políticas, lo encarna. Y, en el conjunto de la acción de la novela Ada, más que los otros milicianos, es la que más impacto tiene sobre el protagonista no nombrado. Su influencia sobre él es muy profunda y de hecho inolvidable y se manifiesta a nivel físico, emocional y psíquico.

El poder físico y sexual que Ada pretende ejercer sobre el protagonista principal comienza desde su primer encuentro en el Aeropuerto Internacional de Malabo cuando cachea a Ángeles, su mujer, de manera tan violenta que podría hablarse de violación. Según cuenta Ángeles a su marido:

Me dijo que me quitara la blusa y me la quité, y el sujetador, y me lo quité, qué vergüenza, amor, mientras con sus rasposas manos me tocaba los sobacos y me acariciaba los senos,... Y luego descendió a los pies, subían sus ásperas y temblorosas manos por mis piernas y por mis muslos hasta bajarme las medias, como si buscase algo, y me quedé paralizada sin poder articular palabra, mientras veía su busto prominente hincharse en una respiración agitada, y me bajó las bragas hasta la rodilla y siguió acariciando mi pubis, luego las nalgas, como si buscase algo arrodillada frente a mí, despacito, voluptuosa, me acariciaba el pubis y metía su dedo extrañamente firme por mi vagina, una vez, otra vez, nunca se sabe qué puede esconder una mujer, dijo mientras masajeaba los pelos de mi pubis. (Ndongo-Bidyogo 40-41)

Vemos aquí que no solamente manda Ada a Ángeles, también toma control de su cuerpo. Ángeles se queda exánime, impotente e indefensa ante la fechoría que Ada lleva a cabo sobre su ser. No se puede negar que con este maltrato de Ángeles, Ada está poniendo de manifiesto el máximo abuso de su posición y del poder. De hecho, Marvin Lewis ha querido interpretar la actuación de Ada como "a symbolic defilement of Spain" (151), o sea, una manera de ven-

${ }^{4}$ Según Ogundipe-Leslie, la teorista nigeriana, creó “... the word 'Stiwanism', instead of feminism, to bypass the combative discourses that ensue whenever one raises the issue of feminism in Africa .... The word feminism' itself seems to be a kind of red tag to the bull of African men. Some say the word by its very nature is hegemonic, or implicitly so. Others find the focus on women in themselves somehow threatening. ... Some who are genuinely concerned with ameliorating women's lives sometimes feel embarrassed to be described as feminist'... 'Stiwa' is my acronym for Social Transformation Including Women in Africa." 22-23. 
garse del antiguo colonizador, lo que encaja perfectamente con la ya mencionada misión de re-africanizar a Guinea Ecuatorial que, como militar, le toca realizar.

Sin embargo, más allá de este acto vengativo, se puede matizar la dinámica de la actuación de Ada un poco más detenidamente, especialmente si consideramos el comentario que hace Ángeles que citamos a continuación:

¿No me habías dicho que no existe el lesbianismo entre las negras, o es que sólo era curiosidad lo que sentía esa horrible mujer? Pero no era sólo curiosidad: ella dijo, sí, que no había visto nunca a una blanca desnuda pero estoy segura de que era lesbiana porque había lascivia en sus movimientos y en sus incipientes jadeos y en su sonrisa y en su pecho hinchado que subía y bajaba al compás de una respiración cada vez más acelerada. (Ndongo-Bidyogo 41).

Este es un hecho y un comentario que una lectura crítica no puede pasar por alto. Los movimientos lascivos junto con la gran satisfacción que Ada cobra manoseando las partes más íntimas del cuerpo de Ángeles ¿acaso no hacen patente una predisposición homoerótica en la miliciana? Al retratar a Ada en estos términos de sugerencia lésbica, e introducir este elemento homoerótico en la obra, Donato Ndongo presenta a la mujer de una manera que anteriormente no ha aparecido en la novela ecuatoguineana. Incluso, en el ámbito más amplio de las literaturas africanas la presentación de la mujer cometiendo actos tan "perversos" se ve más a menudo en escritoras contemporáneas, como Ken Bugul, Calixthe Beyala, Angel Rawiri, consideradas de las más radicales ${ }^{5}$.

Si se toma en cuenta el hecho de que la homosexualidad se considera un elemento crucial que distingue a las feministas africanas de las de Occidente, como se observa en el concepto de womanism desarrollado por la norteamericana Alice Walker y la de la nigeriana Chikwenye Okonjo Ogunyemi, con la figura de Ada se efectúa una subversión a otros dos niveles. No solamente rompe con el orden establecido del sistema patriarcal en el que la única forma de sexualidad aceptable es la heterosexualidad, sino también pone en tela de juicio la práctica en el feminismo africano de hacer caso omiso de la homosexualidad femenina. Bien lo señala Nathalie Etoke cuando dice que: "prominent voices of African feminism or womanism choose not to discuss same-sex love or even acknowledge its existence" (175). Notable entre estas feministas africanas se encuentra a Ifi Amadiume, en cuya opinión el fenómeno de la homosexualidad no entra en las preocupaciones de la mujer africana, o Mary E. Modupe Kolawole, quien afirma:

To the majority of ordinary Africans lesbianism is a non-existent issue because it is a mode of self-expression that is completely strange to their worldview. It is not even an option to millions of African women and can therefore not be the solution as ... many Western or westernized women propose. (15)

Conscientemente o no, y de una manera sutil, con Ada, Ndongo parece llamarle la atención a la crítica feminista africana que niega la presencia de la homosexualidad femenina, denominada "transgresiva" que quizás peque de ingenuidad. Para Julin Everet (1-2):

5 Véase Natalie Etoke, "Mariama Barry, Ken Bugul, Calixthe Beyala, and the Politics of Female Homoeroticism in Sub-Saharan Francophone African Literature" en Research in African Literatures, 2, 2009, pp 173-189. 
queernes is a crucial aspect of African postcolonial literature, firstly because so much of the African cosmologies that inform these texts depends on gender-bending and intersexuality and secondly because, in these texts, stepping outside of established sexual and gender norms is concomitant with political movement, be it that of the revolutionary or of the tyrant. (1-2)

Con este acto que cobra matices lésbicos, por un lado, Ada logra apropiarse para sí el poder dentro del sistema patriarcal en el que transcurren los hechos. Ada infringe los derechos reservados para el abogado protagonista como esposo de Ángeles, y, ocupa su lugar, aunque sea por un momento. Es más, le deja impotente al protagonista no nombrado puesto que, aun a sabiendas de lo ocurrido con su mujer, le es imposible actuar o encontrar reparación. Esto constituye una fuerte apropiación de la posición del hombre negro dentro de una sociedad en la cual la sexualidad del hombre es una de las maneras en que se enfatiza su poder. La posición en que deja Ada al protagonista es similar a la de los vasallos del dictador Trujillo en La fiesta del chivo (1990), novela histórica de Mario Vargas Llosa. Como observamos en la novela histórica los vasallos son incapaces de protestar o buscar reparación cuando el tirano, con impunidad sexualmente posee y hasta viola a sus mujeres e hijas.

Por otro, si se considera el hecho de que Ada (como los otros milicianos) es representante y agente del jefe de estado y de las fuerzas armadas, ella le da forma y encarna al dictador. De acuerdo con esta lógica, es implícito que de manera indirecta es el dictador quien manosea a la mujer del abogado no nombrado. Como nos recuerda Lola Colomina Garrigos, en su estudio sobre el falocentrismo en La fiesta del chivo de Mario Vargas Llosa, "sexo, poder y violación son elementos íntimamente ligados a la figura del dictador." (n.p.) Por consiguiente, con la figura de Ada, Ndongo no simplemente da la vuelta a la tortilla. Ella sirve como arma de doble filo para demostrar la dinámica del poder en que se afirma el poder del dictador a la misma vez que se efectúa la emasculación total del protagonista no nombrado.

La culminación de la dominación física y sexual que ejerce Ada sobre el protagonista principal ocurre en la prisión de Blavis, cuando Ada, rechazada por el protagonista durante su encuentro en la calle, pretende violarlo. Si anteriormente con Ángeles se apoderó de él de manera indirecta, la influencia ejercida esta vez se manifiesta de forma directa. En el espacio de la prisión, la voz de Ada, que desde el encuentro en el aeropuerto y en la calle ha tenido resonancia en la psiquis del abogado, cobra mayor fuerza. Según cuenta "sobre todas destacaba la voz animosa de la miliciana Ada, salid todos, camaradas, que el abogado es mío" (255). La amenaza que sintió el abogado en el encuentro en la calle, razón por la cual se huyó de ella en esa ocasión, se hace realidad. En el último encuentro Ada llega a tocarlo directamente y de manera violenta. Dentro del espacio de la cárcel el abogado que, como describe Otabela, sufre "en sus carnes las más horrendas atrocidades, el cinismo y el sadismo del régimen fascista encabezado por Francisco Macías Nguema” (125), queda exánime (como quedó su mujer en el encuentro en el aeropuerto), mientras Ada manda y domina la situación. Como afirma: "Ahora te tengo a mi disposición, para mí sola, abogado. Puedo hacer contigo lo que me plazca sin que tenga que dar cuenta a nadie" (Ndongo-Bidiyogo 259). Ada desempeña un papel agresivo y activo mientras el abogado se queda pasivo. Además es la única que tiene voz, y habla durante este encuentro siniestro. Tal es la brutalidad efectuada sobre el cuerpo del abogado no nombrado que se ha quedado sin fuerza ni para hablar. 
Se puede argüir, a través de las insinuaciones que hace Ada y de sus acciones que culminan en el encuentro en la prisión, que ella busca tomar el lugar de Ángeles, la mujer del abogado. O sea, dentro del proyecto nacional de re-africanización que, como militar (o encarnación del dictador) le incumbe ayudar a llevar a cabo, la mujer blanca del protagonista sobra, no encaja. Es de notar que parte fundamental del proyecto de re-africanización que la dictadura de Macías impone es la eliminación total de cualquier vestigio del legado colonial español y todo lo que tiene que ver con España. Se pueden explicar el maltrato y la humillación de los blancos españoles como elementos de este proyecto y su eliminación como forma de exorcizar los demonios nacidos de la colonización española. Como hemos demostrado, el personaje que más fuertemente y efectivamente interpreta este proyecto es Ada, y el blanco de sus dardos son el protagonista principal y su mujer española. Además, con su insistencia para tener un encuentro sexual con el abogado, Ada pretende ser "... the woman of color who faithfully and loyally reproduces with the man of color and returns him to his desire, conserved to its proper purpose of national reproduction" (Counihan 164). De llegar a tener una relación sexual con el abogado, habría sustituido a la mujer española del abogado, y su misión se vería completa.

Sin embargo, al contrario de la impotencia física que caracteriza la influencia física que Ada ejerce sobre el abogado que recién ha vuelto a su país, se ve una agitación profunda en lo referente a la influencia emocional y psíquica que Ada provoca en el protagonista narrador. La fuerza de este efecto se evidencia cuando durante uno de los momentos más íntimos que pasa el abogado, cuando su mujer y su hija se habían marchado y se encontraba solo, la imagen de Ada interfiere y se interpone en sus pensamientos. La miliciana provoca toda una gama de emociones en el abogado no nombrado fascinación, duda, desconfianza, angustia, dolor, decepción, frustración, rabia, pavor, repulsión y odio. De modo que es simbólico que en el encuentro en la cárcel, sin poder evitar las náuseas causadas por este revoltijo de emociones, el abogado vomite sobre Ada. Es la única acción que hace el protagonista en ese encuentro. El vómito representa lo no digestible, lo que el cuerpo no acepta y rechaza. Simbólicamente marca un rechazo de todo el poder e influencia que Ada busca ejercer sobre él, y, de hecho todo lo que representa ella.

Con todo lo que venimos señalando, es evidente que el personaje secundario de Ada está lejos de ser el personaje femenino típico de las literaturas africanas que, según Mendogo Minsongui Dieudonné, "es marginada, no tiene nada que decir frente al hombre, que lo decide todo" (211). En todas las instancias en las que aparece en la novela y en sus encuentros con el protagonista no nombrado, es ella que lleva mayor protagonismo en los hechos. Insinúa, comenta, pregunta, ordena, amenaza, cachea, manosea, etc. Tiene voz, actúa, manda, tiene poder.

Pero, aunque Ada dispone de poder, y privilegio ¿hasta qué punto se podría decir que deja de ser víctima, y mujer marginalizada? Según afirma Ogundipe-Leslie, el estatus de la mujer se ve negativamente afectado por los factores siguientes: "The first one is oppression from outside (colonialism and neo-colonialism); the second one is from traditional structures; the third one is her backwardness; the fourth is man; the fifth is her color, her race; and the sixth is herself" (28). Si consideramos a Ada dentro de los parámetros de esta lógica, parece ser que, aparte de ella misma, el hombre tiene mucho que ver con su estatus. Según afirma Daniel Grier, "como apoyadores, o en el caso de Ada, como representante del régimen de 
Macías se le retrata de una manera caricaturesca, pero quizás sean también atrapados dentro de la brutalidad y por eso la única forma de sobrevivirla es colaborar" (74). De este modo, como la encarnación del dictador, Ada aparentemente tiene agencia pero realmente no la tiene. Es solamente una títere a través de la cual se trasmiten los deseos de otro, que responde a los impulsos del dictador, el que realmente manda. Por consiguiente, se puede argüir que como mujer no ha logrado nada. Su afirmación a Ángeles que por ser mujeres "sólo servimos para el placer de los hombres" (Ndongo-Bidyogo, 41) parece corroborar esta posición. En realidad ella está al servicio del dictador quien determina la agenda nacional y para cuyos intereses, como miliciana, le incumbe proteger.

Como queda obvio en la novela, parece ser que conocer al abogado ha despertado en ella cierta necesidad y deseo de él, lo que le lleva a perseguirlo hasta la prisión de Blavis. Según la lógica de lo que venimos citando arriba, su obsesión con el abogado puede interpretarse como su intento de afirmarse como mujer y cumplir con lo que ella percibe ser su responsabilidad darle placer. Cuando la rechaza el abogado, que incluso vomita sobre ella, se efectúa una negación de su estatus como mujer tanto en el contexto de la modernidad como en el tradicional. Parece que ella no sirve para nada. Por ende, la figura de Ada parece reflejar desde los hechos que, a pesar de la aparente liberación de la mujer africana, su posición marginal no ha cambiado, cuando no haya empeorado.

Como observamos en su interacción con los demás, Ada tiene voz, pero casi no dialoga con nadie. Su discurso se caracteriza por órdenes y monólogos, dado que sus interlocutores por distintas razones — por voluntad, temor, incapacidad, o falta de oportunidad — no responden. La primera vez que habla en la novela, según cuenta el narrador: "Ésta, ante tanta ropa femenina le dijo a Ángeles, cogiendo algunas prendas de lencería: Esto me irá bien. ¿Me lo das? Y sin esperar la respuesta se puso bajo las axilas los objetos elegidos" (Ndongo, 27). En el segundo encuentro los pronunciamientos de Ada caen ante la indiferencia del protagonista: "Os habíais distanciado lo suficiente, pero oíste con claridad sus últimas palabras, y ni siquiera volviste los ojos" (Ndongo, 96). Finalmente en la prisión de Blavis "sus palabras producían en mí el efecto contrario al deseado por ella..." (Ndongo, 257). El protagonista hace caso omiso y se pone a pensar en otras cosas: la ausencia de su mujer, las esposas que le hierren las muñecas, la sed que tiene, la rata que le mordía, las cucarachas, hormigas y mosquitos que le molestaban. La mujer aquí se encuentra en una posición de poder y libertad para expresar sus deseos y hacer todo lo que puede para satisfacerlos. A la antigua pero todavía relevante pregunta de Gayatri Spivak de si puede hablar el subalterno ${ }^{6}$, Ada parece responder que sí. Sin embargo, al mismo tiempo hace evidente que es importante lo que se dice en el momento de hablar, y también hace falta que los interlocutores respondan. Es decir, hace falta dialogar, porque, si no, lo que se dice se convierte en ruido.

Mazrui apunta tres deberes interrelacionados para mejorar el destino de las mujeres en cuanto al problema del desequilibrio entre los géneros se refiere: "1) Liberating woman, 2) Centering woman, and 3) Empowering woman. A woman can be at the center without being empowered; a woman can be liberated without being either centered or empowered" (97). A

${ }^{6}$ Véase Gayatri Spivak, "Can the Subaltern Speak?" en Colonial Discourse and Postcolonial Theory, A Reader. Editado por Patrick Williams y Laura Chrisman, New York, Columbia University Press, 1994. 
pesar de tener voz, poder y libertad sexual, la figura de Ada, cuando la vemos por última vez, saliendo de la celda del protagonista central, es la de una despreciada, rechazada, humillada y enloquecida. Esta es una situación que se puede atribuir directamente a su carácter y a la posición que ocupa en la sociedad. Pero quizás más directamente, tiene que ver con sus acciones de ejercer el poder sobre su prójimo de tal manera que lleva al abuso.

En la tradición literaria del norte tanto como la del sur se encuentran casos de mujeres soldados que de un modo u otro juegan un papel importante en el proyecto peculiar de su nación. Tal es el caso de Joan of Arc (inglés), Negra Angustias (mexicana), Nadezhda Durova (rusa), Antonia Tomaszewska (polaca), Yaa Asantewaa (ashanti), por nombrar algunas. Opina Francíková que "However, as scholarship on the history of sexuality makes clear, it is quite unusual for a female soldier to be glorified, even presented as the ideal woman." (357). Aunque las "hazañas" de Ada no alcanzan el mismo nivel de heroísmo que las de las figuras arriba nombradas, es una pieza clave en la supuesta africanización de su país y el proyecto nacional que sus líderes han elegido. Pero, como bien señala Francíková, y se ilustra en Los poderes de la tempestad, la miliciana no se presenta como personaje embellecido. O sea, aunque contribuye de manera significante, y el gobierno cuenta con su aporte al proyecto nacional (aun si es problemático), queda lejos de ser una figura ensalzada.

Con este estudio de Ada y las ocurrencias de la novela una conclusión lógica a que se puede llegar es que la posición de poder que puede tener la mujer no es por derecho propio sino es algo que se le concede el hombre. Realmente, sirve como pretexto para denigrar aún más a la mujer y quitarle cualquier vestigio de dignidad. En verdad, aunque la mujer haya conseguido algunos derechos, el mundo sigue siendo un mundo de hombres y su estatus de víctima sigue vigente, no ha cambiado. Ada se convierte en una rama de los poderes de la tempestad a que hace referencia el título de la novela, y que tiene consecuencias devastadoras tanto para sus conciudadanos como para sí misma como mujer.

\section{REFERENCIAS BIBLIOGRÁFICAS}

Nsüe AngüE, María (2007): Ekomo. Madrid, Casa de África/Sial Ediciones.

Counihan, Clare (2007): "Reading the Figure of Woman in African Literature: Psychoanalysis, Difference and Desire". Research in African Literatures 38.2, p.180-181.

Decosta-Willis, Miriam, (2003): ed. Daughters of the Diaspora: Afra-Hispanic Writers. Kingston, Ian Randle P.

Eтoke, Natalie (2009): "Mariama Barry, Ken Bugul, Calixthe Beyala, and the Politics of Female Homoeroticism in Sub-Saharan Francophone African Literature", Research in African Literatures, 39.2, p. 173-189.

Everet, Julin (2013): "Must La Victime Be Feminine? Postcolonial Violence, Gender Ambiguity, and Homoerotic Desire in Sony Labou Tansi's Le soussigné cardiaque”. Research in African Literatures 44.1, p.1-18.

FrancíKovÁ, DasÁ (2012): "The Queer Story of Katerina Marsalova: The Female Soldier, Ideal Woman, and Masculine Femininity," Gender and History, 24.2, p. 353-367. W

Colomina Garrigos, Lola (2007): "Autoridad discursiva y falocentrismo en La fiesta del chivo de Mario Vargas Llosa". Delaware Review of Latin American Studies. 8.1 n.p. https://www.udel.edu/ LAS/Vol 8-1ColominaGarrigos.html. 
Grier, DANiel (2004): "El exilio y el deseo de cambio en Guinea Ecuatorial: Los poderes de la tempestad de Donato Ndongo Bidyogo", Afrohispanic Review 23.2 p.70-76.

Kolawole, E. M. Mary (1997): Womanism and African Consciousness. Trenton, Africa World Press.

LEWIS, MARvin (2007): An Introduction to the Literature of Equatorial Guinea. Between Colonization and Dictatorship, Columbia, University of Missouri Press.

Mazrui, A. Ali (1993): "The Black Woman and the Problem of Gender: An African Perspective", Research in African Literatures, 24.1, p. 87-104.

Mendogo Minsongui, Dieudonné (1997): "Mujer y creación literaria en Guinea Ecuatorial”, EPOS 13, p. 209-218.

Ndongo Bidyogo, Donato i (1997): Los poderes de la tempestad, Madrid, Morandi.

Ngom, Mbaré (1995): "Relato de una vida y escritura femenina: Ekomo, de María Nsüe Angüe", The Journal of Afro-Latin American Studies and Literatures 3.1, p. 77-92.

Otabela Mewolo, Joseph-Désiré (2003): "Literatura de Guinea Ecuatorial. Sujeto cultural y dictadura: El personaje del abogado en Los poderes de la tempestad de Donato Ndongo Bidyogo", EPOS 19, p.119-128.

Ogundipe-Leslie, Molara (1994): Recreating Ourselves: African Women and Critical Transformations. Trenton, Africa World Press.

Da Silva, I. Meyre (2004): "African Feminists towards the Politics of Empowerment", Revista de Letras, 44.2 p.129-138. 